\title{
SUBJECT INDEX VOLUME 42, 2000
}

Absolute activity, 227-239

Accelerator mass spectrometry (AMS), 127-35, 323-33

Accuracy, 9-16, 221-222

Activity $\left({ }^{14} \mathrm{C}\right), 227-239$

Activity ratio, 227-239

Aerosol, 115-26, 285-94

Allerød, 335-48, 423-35

Alps (French), 423-35

Amino acid separation, 281-4

Antarctica, 51-74

Anthropogenic, 69-80, 81-98, 115-26, 235-48

Aquifer, 235-48

Atmospheric radiocarbon, 370-81, 383-401

Australia, 229-34, 295-308

Austria, 183-198, 285-94

Baltic Sea, 323-33

Basketry, 309-313

Bayesian statistics, 181, 183-98, 199-202

Bellows Dun (Hawaii), 203-17

Benzene, 295-303

Beta counting, 137-50, 281-4

Biomass, 115-26

Biostratigraphic analysis, 301-10

Black Sea, 271-80

Bølling Warm Episode, 137-50

Bones, 257-69

Borehole, 235-48

Calibration, 23-39, 99-114, 137-50, 313-22, 323-33, $335-48,349-53,355-68,383-401,415-22,423-35$, $437-52$

Carbon cycle, $81-98$

Cariaco basin, 335-48, 415-22

Chronometric scale, $1-21$

Clovis culture, $301-10$

Collagen, 281-4

Comparison, 313-22

Corals, 249-56, 383-401

Cosmogenic isotopes, $41-8$

Data base, $41-8$

Decadal time scale, $137-50$

Dendrochronology, 137-50, 335-68

EDTA treatment, 49-52

Foraminifera, 415-22, 437-52

Fossil, 115-26

German pine calibration curve, 335-48

Germany, 349-53, 355-68

Greenhouse gases, 69-80

Groundwater, 99-114
Hawaii, 203-17

Heinrich Event, 437-52

Holocene, 229-34, 423-35

Hydrogeology, 99-114

Iceland Sea, 423-35, 437-52

Ireland, 235-48

Israel, 415-22

Japan, 370-81

Kra, Renee, $\mathrm{v}-\mathrm{xx}(1)$

Lake Gościąż (Poland), 335-48

Lake Holzmaar (Germany), 349-53, 355-68

Lake Lisan (Glacial Dead Sea), 415-22

Lake Meerfelder Maar (Germany), 355-68

Lake Perespilno (Poland), 335-48

Lake Soppensee (Switzerland), 349-53

Lake Suigetsu (Japan), 335-48, 370-81, 415-22

Laschamp, 415-22, 437-52

Lisan aragonite, 415-22

Macrofossils (terrestrial), 335-348

Mammoths, 257-69, 281-4

Maya, 1-21

Mediterranean Sea, 271-80

Mexico, 301-10

Mono Lake, 437-52

New World, 1-21, 257-69

Ocean circulation, 81-98, 249-56, 383-401

Old World, 23-39

Pacific Ocean, 249-56

Paleomagnetic field, 437-52

Particulate carbon (black), 115-26

Phenolic compounds, 219-27

Planktonic reservoir age, $437-52$

Pleistocene, 281-4

Poland, 335-48

Preparative capillary gas chromatography (PCGC), 219-

27

Radiative balance, 69-80, 115-26

Radiocarbon method (history), 127-35, 173-8

Radiocarbon time scale, 137-50, 383-401

Radiometric dating, 257-69

Radionuclides, 151-72

Red Sea, 271-80

Reservoir ages, 271-80

River Ångermanälven (northern Sweden), 323-33 
Sample selection, 203-17

Scandinavian ice sheet, 323-33

Scintillator solution, 295-303

Sediment, 323-33, 335-68, 415-22, 437-52

Shells, 229-34, 271-80

Snail, 229-34

Solar cosmic rays, 151-72

Sonnblick (Austria), 285-94

Spectrum, 295-303

Speleothems, 335-48, 403-14

Stratigraphy, 229-34

Subboreal, 423-35

Sweden, 323-33

Swedish Time Scale, 323-33

Switzerland, 349-53
Tephra, 301-10

Terrestrial biosphere, $69-80$

Thermohaline, 137-50

Time measurement, 257-69

Total dissolved inorganic carbon (TDIC), 235-48

Tufa, 415-22

U-series dating, 383-401, 403-14, 415-22

Varangerfjord, 49-52

Varve, 323-33, 335-48, 349-53, 355-68, 370-81, 403-14 